\title{
Aspectos biopsicossociais relacionados ao consumo de tabaco entre universitários: Uma revisão integrativa
}

\author{
Biopsychosocial aspects related to tobacco consumption among university students: An integrative \\ review
}

Aspectos biopsicosociales relacionados con el consumo de tabaco em estudiantes universitarios:

Una revisión integrativa

Recebido: 18/05/2021 | Revisado: 28/05/2021 | Aceito: 31/05/2021 | Publicado: 04/07/2021

\author{
Thayná de Almeida Alves \\ ORCID: https://orcid.org/0000-0002-7254-6008 \\ Universidade Estadual da Paraíba, Brasil \\ E-mail: thayna.alves@aluno.uepb.edu.br \\ Ana Caroline da Silva Lira \\ ORCID: https://orcid.org/0000-0002-3263-1857 \\ Universidade Estadual da Paraíba, Brasil \\ E-mail: ana.caroline.lira@aluno.uepb.edu.br \\ Clésia Oliveira Pachú \\ ORCID: https://orcid.org/0000-0002-7356-6297 \\ Universidade Estadual da Paraíba, Brasil \\ E-mail: clesiapachu@ hotmail.com
}

\begin{abstract}
Resumo
O tabaco pode gerar dependência química aos seus usuários, principalmente em grupos vulneráveis, como jovens universitários que ao entrar no ensino superior desenvolvem conflitos de princípios empregados pela educação familiar, além da necessidade de inserção social e influência dos amigos para o uso. O presente estudo teve como objetivo analisar os aspectos biopsicossociais relacionados ao consumo de tabaco entre universitários. Para atingir esse objetivo, realizou-se uma revisão integrativa da literatura em que foram utilizadas as bases de dados SciELO Brasil, SciELO Saúde Pública, SciElo, OneFile e Scopus, aplicando os termos "aspectos biopsicossociais", "consumo de produtos derivados do tabaco", "consumo de tabaco", "tabagismo", "universitário". A partir dessa busca, empregou-se os critérios de inclusão e exclusão, restando dez artigos na amostra final de uma totalidade de 3.105 artigos da busca inicial. Foi observado que os universitários são vulneráveis à utilização de tabaco devido a fatores como: a influência social, a pressão e as situações de ansiedade geradas pela universidade. O consumo por jovens é marcado pelo uso do tabaco com outras drogas, geralmente o álcool, provocando danos à saúde. Conclui-se que os estudantes de área da saúde são mais ativos no consumo, mesmo tendo conhecimento dos riscos causados pelo tabaco. Palavras-chave: Consumo de produtos derivados do tabaco; Angústia psicológica; Impactos na saúde; Estudantes; Fatores sociológicos.
\end{abstract}

\begin{abstract}
The tobacco can generate chemical addiction to its users, especially in vulnerable groups such as university students which upon entering university may develop conflicts of principles employed by family education, besides the need for social insertion and the influence of friends The purpose of this study is the analysis of the biopsychosocial aspects related to tobacco consumption among college students. To reach this objective, an integrative literature review was carried out using the SciELO Brasil, SciELO Saúde Pública, SciElo, OneFile and Scopus databases. Using the terms "aspectos biopsicossociais", "consumo de produtos derivados do tabaco", "consumo de tabaco", "tabagismo", "estudante universitário". The inclusion and exclusion criteria were observed on 3.105 articles in which ten of them made up the final sample. It was observed that college students are vulnerable to tobacco use due to factors such as social influence, psychological pressure, and anxious situations generated by the university. Tobacco consumption by young people is marked by the use of other drugs, usually alcohol, causing harm to health. It is concluded that health students are more active in this consumption, despite knowing the risks caused by tobacco.
\end{abstract}

Keywords: Consumption of tobacco derived products; Psychological anguish; Health impacts; Students; Sociological factors. 


\section{Resumen}

El tabaco puede generar dependencia química a sus usuarios, principalmente en grupos vulnerables, como jóvenes universitarios, que al entrar en la enseñanza superior desarrollan conflictos con los principios recibidos en la educación familiar, además de la necesidad de inserción social e influjo de los amigos para el uso. El presente trabajo tiene como objetivo analizar los aspectos biopsicosociales que están relacionados al consumo de tabaco entre los universitarios. Para alcanzar este objetivo, fue realizado un repaso integrativo de la literatura, en lo que utilizamos las bases de dados Scielo Brasil; Scielo Saúde Pública; OneFile e Scopus, con el empleo de los términos "aspectos biopsicosociales"; "consumo de productos derivados de tabaco"; "consumo de tabaco"; "tabaquismo" e "universitario". Basados en esta búsqueda, recurrimos a los criterios de inclusión y exclusión, en la muestra final se quedarán diez artículos de una totalidad de 3.105 iniciales. Se observó que los universitarios son vulnerables a la utilización de tabaco debido a factores como: influjo social, depresión y situaciones de ansiedad generadas por la universidad. El consumo por los jóvenes está marcado por el consumo junto a otras drogas, generalmente el alcohol, trayendo danos a la salud. Se concluye que los estudiantes del área de salud son los más enérgicos en el consumo, mismo teniendo conocimiento de los riscos causados por el tabaco.

Palabras claves: Consumo de productos derivados del tabaco; Distrés psicológico; Impactos en la salud; Estudiantes; Factores sociológicos.

\section{Introdução}

O tabaco, Nicotiana tabacum, tem como princípio ativo a nicotina, capaz de gerar dependência química em quem a utiliza. Observa-se que, tendo como base o tabaco, são produzidos diversos produtos, como o cigarro, charuto, cachimbo, narguilé, rapé, fumo-de-rolo e o cigarro eletrônico, sendo todos esses nocivos à saúde (Brasil, 2016).

Nesse contexto, considera-se o tabagismo uma doença crônica decorrente da dependência à nicotina, encontrada nos produtos à base de tabaco. O tabagismo de acordo com a Classificação Estatística Internacional de Doenças e Problemas Relacionados à Saúde (CID-10), classifica-se no grupo de transtornos mentais e de comportamento, por consistir no uso de substâncias psicoativas (Instituto Nacional de Câncer [INCA], 2021).

De acordo com a Organização Pan-Americana de Saúde (2019), o tabaco, encontra-se responsável pela morte de mais de 8 milhões de pessoas ao ano, sendo mais de 7 milhões resultantes do uso direto do produto, sendo outros 1,2 milhões decorrentes do fumo passivo. O tabagismo representa a maior causa de adoecimento e mortes precoces no mundo. No Brasil, os dados são igualmente alarmantes, 156.216 mortes por ano, principalmente, por doenças cardiovasculares, Doença Pulmonar Obstrutiva Crônica (DPOC), câncer, pneumonia, tabagismo passivo e Acidente Vascular Cerebral (AVC) (Drope \& Schluger, 2018; Pinto et al., 2017).

Nesse sentido, a entrada no ensino superior, graduação, traz consigo uma sucessão de eventos, alguns deles inesperados, acarretando uma nova realidade para os estudantes em termos cognitivos e emocionais. Os jovens universitários constituem um grupo vulnerável, pois com o ingresso no centro universitário ocorrem incertezas e hesitações de princípios, crenças e posicionamentos ensinados pela família durante processo de desenvolvimento da educação. Tais questionamentos podem refletir em um novo comportamento, por consequência, podem influenciar em um estado de saúde percebido de forma positiva ou negativa. Quando o indivíduo não possui habilidade de lidar com essas mudanças elas podem se tornar uma motivação de sofrimento psíquico, podendo se destacar a ansiedade e estresse (Brito, Gordia \& Quadros, 2016).

Ainda cabe destacar que, além de toda vulnerabilidade decorrente das diversas mudanças ao adentrar no meio universitário, há maior autonomia, novas situações a serem vivenciadas e o desafio de conhecer seus limites. Fazendo-se preciso considerar também a influência do fator social nesse processo, dada a realização de festas universitárias e a pressão imposta pelos colegas para o uso de drogas como forma de inserção social (de Assis Trindade, Diniz \& Sá-Junior, 2018; Weitzman, Nelson \& Wechsler, 2003). 
Segundo o Ministério da Saúde (2011), cerca de 36,7\% dos jovens brasileiros fazem consumo de álcool e 23,9\% fumam cigarro. Deste modo, a segunda droga lícita mais utilizada no ambiente universitário é o tabaco, principalmente facilitado pelo apoio legal de sua fabricação, venda e consumo, especialmente no Brasil (Fernandes, 2017).

Observando as políticas de saúde voltadas para o controle do tabagismo, especialmente entre jovens, encontra-se uma iniciativa mundial de 180 países chamada Convenção-Quadro para o Controle do Tabaco (CQCT). Esta norteia, a nível nacional, a Política Nacional de Controle do Tabaco (PNCT). De fato, no Brasil, essas ações têm mostrado grandes resultados, obtendo, por exemplo, uma redução percentual de 22,8\% em 2008 para 18,7\% em 2013, de homens fumantes com mais de 18 anos, e as mulheres também apresentaram uma redução de 13,8\% para 10,8\% (Brasil, 2015).

Ainda, segundo o mesmo autor, apesar da avaliação da taxa de cessação do tabaco ter sido positiva entre os homens em 2013, se for comparada as faixas etárias de menores de 25 anos (32,6\%) e maiores de 25 anos (55,1\%), observa-se que essa taxa ainda se apresenta menor entre os mais jovens.

Dessa forma, pretende-se responder à pergunta: Como os aspectos biopsicossociais se relacionam ao consumo de tabaco entre universitários? Este estudo objetivou analisar os aspectos biopsicossociais relacionados ao consumo de tabaco entre universitários por meio de uma revisão integrativa.

\section{Metodologia}

O presente estudo consiste em uma revisão integrativa da literatura acerca dos aspectos biopsicossociais relacionados ao consumo de tabaco entre universitários, de instituições de ensino públicas ou privadas, em revistas indexadas. Nesse sentido, a facilidade e amplitude que os meios digitais oferecem atualmente tem favorecido os pesquisadores na tentativa de atualização da literatura existente (Brevidelli \& Domenico, 2006).

Para coleta de dados, buscou-se artigos da literatura científica fazendo uso dos periódicos disponíveis nas bases de dados SciELO Brasil, SciELO Saúde Pública, SciElo (Crossref), OneFile (Gale) e Scopus (Elsevier).

Foram utilizados os termos "Aspectos biopsicossociais", "Consumo de produtos derivados do tabaco", "Consumo de tabaco", "Tabagismo", "Universitário", e junto a esses, o operador booleano "AND".

Os critérios de inclusão utilizados foram: artigos nos idiomas português, inglês e espanhol, publicados no período de 2016 a 2021 nas referidas bases de dados e que apontassem para a resposta da pergunta norteadora.

Como critérios de exclusão foram excluídos os artigos que apresentaram um público diferente do preterido na presente pesquisa, bem como, aqueles que estavam repetidos ou que não atendiam ao critério temporal definido para estudo.

\section{Resultados}

A busca estratégica na base de dados pode ser exposta pelo fluxograma descritor dos resultados obtidos, de acordo com o fluxograma PRISMA (Figura 1). 
Figura 1 - Fluxograma de seleção das publicações acerca dos aspectos biopsicossociais relacionados ao consumo de tabaco entre universitários.
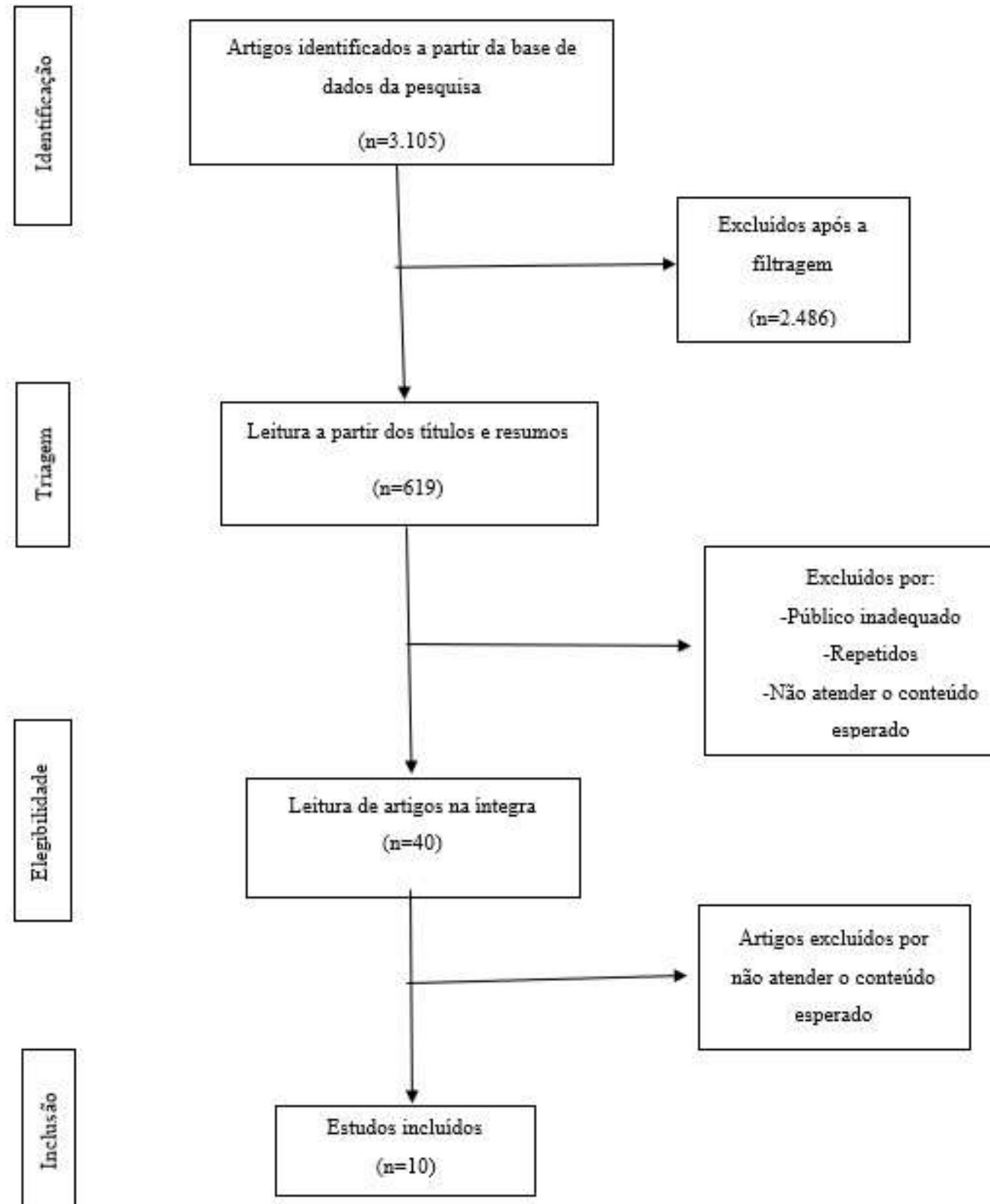

Artigos excluidos por

não atender o conteúdo

experado

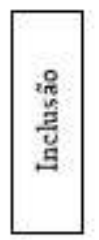

Fonte: Autores (2021).

A pesquisa inicial obteve 3105 artigos que, após realização da aplicação dos filtros, foram reduzidas a 2486. Foram então excluídos aqueles que não pertenciam às bases de dados definidas na presente pesquisa, restando apenas 619. Realizouse a leitura dos títulos e resumos e foram capturados 40 artigos para leitura na íntegra. Por fim, após essa leitura, com base na pergunta norteadora deste estudo, restaram 10 artigos. 
Tabela 1 - Matriz da coleta de dados acerca dos aspectos biopsicossociais relacionados ao consumo de tabaco entre universitários de instituições de ensino públicas e privadas.

\begin{tabular}{|c|c|c|c|c|c|}
\hline$N$ & ملسئ Tít & Awtores & Método & Base & Princinais conclusñes \\
\hline A1 & $\begin{array}{l}\text { Lifestyle of students } \\
\text { admitted to a Nutrition } \\
\text { and Dietetics program. } \\
\text { Bogotá D.C., 2010-2011 }\end{array}$ & $\begin{array}{l}\text { Becerra-Bulla, } \\
\text { Fabiola ; Zarate, } \\
\text { Melier Vargas- }\end{array}$ & $\begin{array}{l}\text { Observacion } \\
\text { al, } \\
\text { transversal e } \\
\text { descritivo }\end{array}$ & Scopus & $\begin{array}{l}\mathrm{Na} \text { juventude ocorrem ajustes sociais e de } \\
\text { personalidade, sendo uma fase de ansiedade. A } \\
\text { universidade é facilitadora do aumento do consumo. }\end{array}$ \\
\hline A2 & $\begin{array}{l}\text { Risk perception for } \\
\text { alcohol and tobacco } \\
\text { consumption in } \\
\text { Saltillo's Health } \\
\text { Sciences students }\end{array}$ & $\begin{array}{l}\text { ANGULO, } \\
\text { P.G., Martínez, } \\
\text { E.K.H.; Puente, } \\
\text { L.A.R.; Vargas, } \\
\text { R.C.; Mendoza, } \\
\text { J.S.; Martínez, } \\
\text { J.U.C. }\end{array}$ & $\begin{array}{l}\text { Descritivo e } \\
\text { correlaciona } \\
1\end{array}$ & Scopus & $\begin{array}{l}\text { A idade de início do uso de tabaco era } 17 \text { anos, uma } \\
\text { idade de busca sentir como integrantes da sociedade. } \\
\text { Geralmente é onde inicia a vida acadêmica, obtendo a } \\
\text { influência de novos amigos que os levam a adquirir } \\
\text { comportamentos, como o uso de tabaco }\end{array}$ \\
\hline A3 & 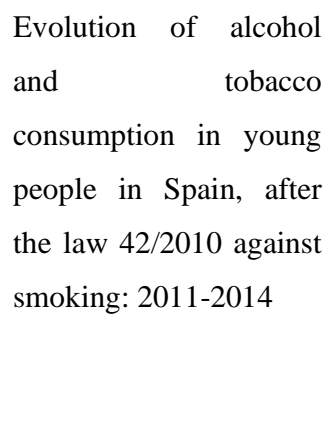 & $\begin{array}{l}\text { Muños, P.M.R; } \\
\text { Torres, J.M.C.; } \\
\text { Lopezosa, P.H.; } \\
\text { Cuenca, A.I.C.; } \\
\text { Borrego, } \\
\text { M.A.R. }\end{array}$ & $\begin{array}{l}\text { Descritivo e } \\
\text { transversal }\end{array}$ & Scopus & $\begin{array}{l}\text { Grande parte dos jovens são policonsumidores, o } \\
\text { álcool é o mais consumido e um fator de risco para o } \\
\text { fumo e para o aumento de cigarros consumidos. Na } \\
\text { juventude o uso é por diversão, novas experiências, } \\
\text { tentativa de pertencer a um grupo e adquirir } \\
\text { identidade pessoal. Mesmo que o uso se dê por } \\
\text { cigarro eletrônico, muito popular entre os jovens, } \\
\text { acarreta danos. }\end{array}$ \\
\hline A4 & $\begin{array}{l}\text { Práctica de actividad } \\
\text { física, consumo de } \\
\text { tabaco y alcohol y sus } \\
\text { efectos en la salud } \\
\text { respiratória de los } \\
\text { jóvenes universitários }\end{array}$ & $\begin{array}{l}\text { Muñoz, S.P.; } \\
\text { Fernández- } \\
\text { Luna, A. }\end{array}$ & $\begin{array}{l}\text { Quantitativo } \\
\text {, descritivo } \\
\text { e transversal }\end{array}$ & Scopus & $\begin{array}{l}\text { O uso de tabaco está ligado ao prejuízo da função } \\
\text { respiratória, o que piora com a quantidade de maços } \\
\text { por dia, trazendo danos à saúde, por exemplo a } \\
\text { DPOC, muito presente nos universitários. }\end{array}$ \\
\hline A5 & $\begin{array}{l}\text { Estilos de vida de } \\
\text { estudiantes de } \\
\text { enfermeria de una } \\
\text { universidad publica } \\
\text { colombiana }\end{array}$ & $\begin{array}{l}\text { Rodriguez- } \\
\text { Gazquez, M. } \\
\text { L.A; Chaparro- } \\
\text { Hernandez, } \\
\text { S.; Gonzalez- } \\
\text { Lopez, J.R }\end{array}$ & $\begin{array}{l}\text { Trasversal } \\
\text { descritivo }\end{array}$ & OneFile & $\begin{array}{l}\text { Estudantes de enfermagem mostraram bastante } \\
\text { contato com o tabaco. Apesar de possuírem } \\
\text { conhecimento, negligenciam a cuidado consigo, } \\
\text { causando danos à saúde e futura prática profissional. } \\
\text { Esses ainda possuem pior qualidade de sono, níveis de } \\
\text { estresse, com tipos de comportamento inadequados e } \\
\text { com mais tempo de curso }\end{array}$ \\
\hline A6 & $\begin{array}{l}\text { Validação do the } \\
\text { alcohol, moking and } \\
\text { substance involvement } \\
\text { screening test (assist) } \\
\text { em estudantes } \\
\text { universitários }\end{array}$ & $\begin{array}{l}\text { Mostardinha, } \\
\text { A.R; Bártolo, } \\
\text { A.; Bonifácio, } \\
\text { J. ; Pereira, A. }\end{array}$ & $\begin{array}{l}\text { Validação } \\
\text { transversal }\end{array}$ & Scopus & $\begin{array}{l}\text { O uso social do tabaco é popular no meio acadêmico, } \\
\text { podendo impulsionar ainda mais a iniciação entre os } \\
\text { universitários. Este consumo traz riscos à saúde, a } \\
\text { médio e longo prazo, mesmo que assintomático, que } \\
\text { pode interferir na identificação desses problemas de } \\
\text { saúde. }\end{array}$ \\
\hline
\end{tabular}




\begin{tabular}{|c|c|c|c|c|c|}
\hline A7 & $\begin{array}{l}\text { Prevalência e fatores } \\
\text { associados ao consumo } \\
\text { de álcool e tabaco entre } \\
\text { estudantes de medicina } \\
\text { no Nordeste do Brasil }\end{array}$ & $\begin{array}{l}\text { PINHEIRO, } \\
\text { Marcelo de } \\
\text { Almeida et al. }\end{array}$ & Analítico & SciELO & $\begin{array}{l}\text { O consumo do álcool associa-se do ao tabaco. Apesar } \\
\text { dos estudantes da área de saúde terem conhecimento } \\
\text { dos danos, o período acadêmico é propício ao início } \\
\text { do fumo por aspectos associado ao ambiente } \\
\text { universitário }\end{array}$ \\
\hline A8 & $\begin{array}{l}\text { Percepción de riesgo } \\
\text { respecto al consumo de } \\
\text { tabaco en jóvenes } \\
\text { universitarios cubanos }\end{array}$ & $\begin{array}{l}\text { Fernández- } \\
\text { Castillo, } \\
\text { Pérez, O. } \\
\text { Torres, L. } \\
\text { Peña, A.; } \\
\text { Grau, R. }\end{array}$ & $\begin{array}{l}\text { Descritivo, } \\
\text { não } \\
\text { experimenta } \\
1 \text { e } \\
\text { transversal }\end{array}$ & SciELO & $\begin{array}{l}\text { Os universitários reconhecem os malefícios do } \\
\text { tabagismo no ponto de vista sociais, físico e } \\
\text { psicológico. Quando se trata do fator motivacional - } \\
\text { comportamental existe uma dispersão já que se } \\
\text { considera personalidade, caráter e individualização da } \\
\text { percepção de risco. }\end{array}$ \\
\hline A9 & $\begin{array}{l}\text { Consumo de drogas en } \\
\text { estudiantes de } \\
\text { enfermería }\end{array}$ & $\begin{array}{l}\text { Garrido- } \\
\text { González, I.; } \\
\text { Bugarín- } \\
\text { González, R.; } \\
\text { Machín- } \\
\text { Fernández, A. J. }\end{array}$ & $\begin{array}{l}\text { Observacion } \\
\text { al, } \\
\text { descritivo e } \\
\text { transversal }\end{array}$ & Scopus & $\begin{array}{l}\text { Os padrões de consumo do tabaco no meio } \\
\text { universitário estão semelhantes ao da população geral. }\end{array}$ \\
\hline A10 & $\begin{array}{l}\text { Descripción de las } \\
\text { conductas de salud en } \\
\text { un grupo de estudiantes } \\
\text { universitarios de Lima }\end{array}$ & Becerra,S. & $\begin{array}{l}\text { Analítico e } \\
\text { descritivo }\end{array}$ & SciELO & $\begin{array}{l}\text { O aumento do consumo de tabaco e a diminuição de } \\
\text { idade de início é um aspecto relevante e pode } \\
\text { representar um grande risco para a saúde podendo até } \\
\text { desenvolver a dependência. }\end{array}$ \\
\hline
\end{tabular}

Fonte: Autores (2021).

A partir do estudo dos 10 artigos selecionados no presente estudo foi possível realizar a extração de todas as informações pertinentes para responder à pergunta do presente estudo (Tabela 1).

Os artigos selecionados integravam, em sua maioria, a Scopus (6), seguido da Scielo (3) e, por fim, a OneFile (1). A maior parte apresenta caráter descritivo, sendo somente um realizado no Brasil.

\section{Discussão}

O consumo de tabaco vem aumentando de forma abusiva. Essa dependência tem se tornando um grande problema por estar acontecendo cada vez mais precocemente na adolescência, afetando a cada dia um público mais jovem e o período de transição para universidade tem sido apontado como uma fase de grande vulnerabilidade para o consumo dessas substâncias. A inserção na instituição universitária, tida pelos jovens como uma conquista de autonomia, representa um período em que muitos se sentem pressionados com a responsabilidade de cuidar de si e do próprio futuro. O ambiente acadêmico se torna capaz de proporcionar aos estudantes oportunidades únicas, incluindo aprendizados, formação de grupos, construção de novos princípios e compartilhamento de experiências (A2, A7 e A10). Desse modo, acontece o incentivo e encorajamento de algumas práticas e costumes por colegas (Silva, 2017).

Nesse sentido, Araújo, Milhornem, Pereira e Silva Junior (2019) constatou que estudantes de medicina do $1^{\circ}$ ao $6^{\circ}$ ano de curso por apresentarem o maior conhecimento dos malefícios, especialmente nos acadêmicos do último ano, não se relacionava a maior intenção de interromper o consumo ou a menor frequência deste. De fato, os universitários não são um público leigo acerca dos malefícios que o consumo de tabaco traz ao organismo (A5, A7 e A8). Especialmente os estudantes da 
saúde, mesmo sendo conhecedores dos riscos, dado o envolvimento do meio universitários e todos os problemas que carrega, não fazem disso um motivo para abandonar o uso.

Uma pesquisa realizada com estudantes de psicologia acerca do uso, e os fatores relacionados a este, de Álcool e outras Substâncias Psicoativas (SPAs) observou que, de acordo com os participantes, o álcool atua como potencializador da vontade de fumar. Isso reforça a ideia do álcool como fator de risco para iniciação do fumo, tornando esses jovens ainda mais expostos ao policonsumo (A3 e A7) (Pires, Farinha, Pillon \& Santos, 2020).

Diversos fatores estão associados ao uso de tabaco por universitários, dentre eles, a ansiedade, a necessidade de inserção social, a influência dos amigos, o estresse e a carga acadêmica exaustiva (A1, A2, A3, A5, A6, A7 e A8). De modo semelhante foi observado em um estudo com universitários da área da saúde que relataram a ocorrência do consumo de tabaco. Esta prática ocorria, geralmente, junto ao álcool em ambientes festivos, quando estavam na presença de outro fumante. Também acontecia, na apresentação de sentimentos de tristeza e ansiedade e pelo estímulo de amigos fumantes (Junior, Santana \& Sousa, 2016).

Quanto maiores os indícios de depressão, ansiedade e estresse, maior o uso de tabaco visando reduzir os sintomas gerados pela vida acadêmica (A5, A6 e A7). A prevalência do uso da substância em questão por estudantes da área da saúde parece ser superior ao uso por universitários de outras áreas, podendo estar relacionados ao fato de demandas intensas, tais como estágios de longa duração, contato direto com problemas de saúde e óbitos. Bem como, a convivência com colegas que consomem o cigarro/tabaco e influencia o uso (Beneton, Schmitt \& Andretta, 2021).

$\mathrm{O}$ uso do cigarro eletrônico (CE) tem se tornado popular entre os jovens. No entanto, apesar de ter sido criado como alternativa para o abandono ao tabagismo, não há evidências claras que sustentem a eficácia desse objetivo (A3). Apesar dos dados sugerirem benefícios no uso do cigarro eletrônico para redução do fumo, são inconclusivos e carregam uma preocupação maior: o uso do CE serve como porta de entrada para o tabagismo em jovens (Oliveira, 2016).

O tabaco traz danos à saúde, especialmente, vários tipos de cânceres. Pode-se citar, também, o prejuízo ao sistema circulatório, com o aumento da pressão arterial, diminuição do oxigênio circulante, formação de trombos, aterosclerose e produção da carboxihemoglobina. No sistema respiratório, o consumo de tabaco, lesiona as vias aéreas que causam principalmente bronquites crônicas, enfisema pulmonar e, assim, a doença pulmonar obstrutiva crônica (DPOC). Entretanto, os danos não se resumem somente a esses sistemas, ocasionando ainda impotência sexual, infertilidade, catarata e tantos outros danos (A4, A6). Os jovens, por sua vez, apesar de conhecerem os riscos, como exemplo, a maior parte dos universitários, não medem os impactos que o uso a longo prazo, confiando na sua jovialidade (Rosemberg, 2003; INCA, 2021).

Os impactos do tabagismo não se refletem apenas na sua vida pessoal, em especial na área da saúde (A5, A7). Um estudo realizado na Itália com estudantes de medicina tabagistas revelou que, como profissionais, esses indivíduos eram menos dispostos a conceder o tratamento e aconselhamento para abandono do uso a seus pacientes, impactando também na saúde destes. Considerando que a universidade representa ambiente de iniciação da vida profissional, torna-se importante também que este ambiente ofereça suporte educativo e exemplo à comunidade acerca da redução do tabagismo, sendo frente nessa iniciativa (Armstrong, Veronese, George, Montroni \& Ugolini, 2017).

Segundo o INCA (2021), o percentual de tabagismo na população acima de 18 anos no Brasil tem apresentado uma expressiva queda em consequência das Políticas de Controle do Tabagismo implementadas, foi observado no ano de 2003, pela Pesquisa Mundial de Saúde (PMS) que o percentual era de 22,4\%. Dados de 2019 obtidos pela Pesquisa Nacional de Saúde (PNS) apontam o percentual total de adultos fumantes é de 12,6\%. (A9). Já o percentual de prevalência do uso de tabaco nos últimos 30 dias entre universitários foi de 21,6\%. Assim, fica demonstrado que os padrões de consumo são semelhantes aos da população geral nessa faixa etária, sendo alguns deles mais elevados. 


\section{Considerações Finais}

Percebe-se que o ambiente universitário possibilita diversas oportunidades para o consumo do tabaco, a exemplo das frequentes festas universitárias e ainda pelo estresse, ansiedade e depressão. Assim, apresenta-se potencializado pelos desafios da vida acadêmica, como a quantidade de atividades a serem desenvolvidas e a maior responsabilidade que esta fase exige.

É relevante ressaltar que a literatura aponta que os estudantes da área da saúde fazem mais uso de substâncias e podem fazê-lo com o intuito de lidar com suas dificuldades na vida universitária. Esses ainda detêm um maior conhecimento acerca dos danos que o uso de tabaco pode causar, sendo que o conhecimento dos malefícios do tabaco não se relaciona ao abandono do consumo. Possivelmente, esses jovens podem apresentar dificuldades em lidar com pacientes que buscam se abster do tabaco.

Além disso, quando se fala em tabaco se faz importante considerar, além do cigarro, as suas diversas formas, a exemplo do cigarro eletrônico e do narguilé, têm se tornado populares entre os jovens. Apesar do cigarro eletrônico trazer uma proposta de tratamento para o abandono ao tabaco, os estudos científicos são inconclusivos e apontam que este pode servir como porta de entrada para o tabagismo.

Ademais, destaca-se que a universidade representa um ambiente de suporte educativo, devendo defender e dar exemplo acerca da redução do tabagismo, em especial, entre seus membros.

Portanto, entende-se por imperativo firmar a necessidade de produção de futuros estudos como forma de implementação/implantação da prática de prevenção à dependência química e promoção de saúde no ambiente universitário privilegiando a qualidade de vida de adolescentes e jovens.

\section{Referências}

Armstrong, G. W., Veronese, G., George, P. F., Montroni, I., \& Ugolini, G. (2017). Avaliação dos hábitos, atitudes e educação do tabaco entre estudantes de medicina nos Estados Unidos e na Itália: uma pesquisa transversal. Journal of Preventive Medicine and Public Health, 50 (3), 177.

Araújo, R. D. S., Milhomem, Y. D. O., Pereira, H. F. S., \& Silva Junior, J. L. R. D. (2019). Fatores relacionados ao consumo do narguilé entre estudantes de medicina. Jornal Brasileiro de Pneumologia, 45(5).

Beneton, E. R, Schmitt, M., \& Andretta, I. (2021). Sintomas de depressão, ansiedade e estresse e uso de drogas em universitários da área da saúde. Revista da SPAGESP, 22(1):145-59.

Brasil (2016). Ministério da Saúde. Glossário temático: fatores de proteção e de risco de câncer. Secretaria-Executiva. Secretaria de Atenção à Saúde. Ministério da Saúde.

Brasil (2015). Ministério da Saúde. Ministério da Saúde celebra dez anos de iniciativa para combater o fumo. Ministério da Saúde.

Brasil (2011). Ministério da Saúde. Pesquisa de Conhecimentos, Atitudes e Práticas na População Brasileira de 15 a 64 anos 2008 . Brasília: Ministério da Saúde.

Brevidelli, M. M., \& Domenico, E. B. L. (2006). Guia prático para docentes e alunos da área de saúde. Trabalho de conclusão de curso. Iátria.

Brito, B. J., Gordia, A. P., \& Quadros, T. M. B. (2016). Estilo de vida de estudantes universitários: estudo de acompanhamento durante os dois primeiros anos do curso de graduação. Medicina (Ribeirão Preto), 49(4), 293-302.

de Assis Trindade, B. P., Diniz, A. V., \& Sá-Júnior, A. R (2018). Uso de drogas entre estudantes universitários: uma perspectiva nacional. Revista de Medicina e Saúde de Brasília, 7 (1).

Drope, J., \& Schluger, N. W. (Eds.). (2018). O atlas do tabaco. Georgia: Sociedade americana de câncer.

Fernandes, T. F., Monteiro, B. M. D. M., Silva, J. B. M., Oliveira, K. M. D., Viana, N. A. O., Gama, C. A. P. D., \& Guimarães, D. A. (2017). Uso de substâncias psicoativas entre universitários brasileiros: perfil epidemiológico, contextos de uso e limitações metodológicas dos estudos. Cadernos Saúde Coletiva, 25(4), 498-507.

Instituto Nacional do Câncer - INCA (2021). Dados e números da prevalência do tabagismo. Ministério da Saúde.

Instituto Nacional do Câncer - INCA (2021). Tabagismo. Ministério da Saúde.

Junior, G. A, Santana, M. L, \& Sousa, T. H. S. (2016). A Exposição do Uso do Tabaco e a Condição de Saúde do Universitário. Brazilian Journal of Forensic Sciences, Medical Law and Bioethics, 5(2):183-97. 
Research, Society and Development, v. 10, n. 7, e11210716250, 2021

(CC BY 4.0) | ISSN 2525-3409 | DOI: http://dx.doi.org/10.33448/rsd-v10i7.16250

Oliveira, L. A. (2016). Experimentação e uso de cigarro eletrônico e narguilé entre universitário. Dissertação de Mestrado. Faculdade de Medicina, Universidade Federal de Goiás. Goiania, Brasil.

Organização Pan-Americana da Saúde - OPAS (2019). Folha informativa - Tabaco. Organização Mundial de Saúde.

Pinto, M., Bardach, A., Palacios, A., Biz, A. N, Alcaraz, A., Rodríguez, B., Augustovski, F., et al. (2017). Carga de doença atribuível ao uso do tabaco no Brasil e potencial impacto do aumento de preços por meio de impostos. Documento técnico IECS $\mathrm{N}^{\circ} 21$. Buenos Aires: Instituto de Efectividad Clínica y Sanitaria.

Pires, I. T. M., Farinha, M. G., Pillon, S. C., \& Santos, M. A. D. (2020). Uso de Álcool e outras Substâncias Psicoativas por Estudantes Universitários de Psicologia. Psicologia: Ciência e Profissão, 40.

Rosemberg, J. N. (2003). Droga Universal. Brasil.

Silva, P. D. C. (2017). Investigar o consumo de substâncias psicoativas entre universitários ingressantes de uma instituição pública. Trabalho de conclusão de curso. Universidade Federal de Mato Grosso. Cuiabá, Brasil.

Weitzman, E. R., Nelson, T. F., \& Wechsler, H. (2003). Começando a beber em excesso na faculdade: as influências da pessoa, do grupo social e do ambiente. Jornal da saúde do adolescente, 32 (1), 26-35. 